\title{
UNKNOTTING TUNNELS, BRACELETS AND THE ELDER SIBLING PROPERTY FOR HYPERBOLIC 3-MANIFOLDS
}

\author{
COLIN ADAMS ${ }^{凶}$ and KARIN KNUDSON
}

(Received 11 August 2011; accepted 6 September 2012; first published online 7 June 2013)

Communicated by C. Hodgson

\begin{abstract}
An unknotting tunnel in a 3-manifold with boundary is a properly embedded arc, the complement of an open neighborhood of which is a handlebody. A geodesic with endpoints on the cusp boundary of a hyperbolic 3-manifold and perpendicular to the cusp boundary is called a vertical geodesic. Given a vertical geodesic $\alpha$ in a hyperbolic 3-manifold $M$, we find sufficient conditions for it to be an unknotting tunnel. In particular, if $\alpha$ corresponds to a 4-bracelet, 5-bracelet or 6-bracelet in the universal cover and has short enough length, it must be an unknotting tunnel. Furthermore, we consider a vertical geodesic $\alpha$ that satisfies the elder sibling property, which means that in the universal cover, every horoball except the one centered at $\infty$ is connected to a larger horoball by a lift of $\alpha$. Such an $\alpha$ with length less than $\ln (2)$ is then shown to be an unknotting tunnel.
\end{abstract}

2010 Mathematics subject classification: primary 57M50.

Keywords and phrases: hyperbolic 3-manifold, unknotting tunnel.

\section{Introduction}

An unknotting tunnel $\alpha$ in a manifold $M$ with boundary is an arc properly embedded in the manifold such that $M-N(\alpha)$ is a handlebody. Given a knot or link $K$ in $S^{3}$, an $\operatorname{arc} \alpha$ that intersects $K$ in its endpoints is said to be an unknotting tunnel if it is an unknotting tunnel when restricted to the exterior of $K$.

The tunnel number of a manifold is the least number of properly embedded arcs such that the complement of an open regular neighborhood of the arcs is a handlebody. Every compact orientable manifold with boundary has a finite tunnel number associated with it, but here, we will be dealing with manifolds of tunnel number one.

We consider finite volume orientable hyperbolic manifolds with one or two cusps. Examples include hyperbolic knot or link complements. If $M$ is such a manifold,

(c) 2013 Australian Mathematical Publishing Association Inc. 1446-7887/2013 \$16.00 
then by [10], the hyperbolic structure is unique, and there exists a projection map $p: H^{3} \rightarrow M$ that generates the hyperbolic structure. Cusps then lift to collections of horoballs in hyperbolic 3-space, and geodesics with both ends going out the cusps lift to collections of geodesics connecting horoballs. Such geodesics are called vertical geodesics and are candidates for being unknotting tunnels when the interiors of disjoint cups are removed.

In [1], it was proved that for two-cusped hyperbolic 3-manifolds, all unknotting tunnels are vertical geodesics, and further, if the length of such a geodesic is defined as the length outside a maximal cusp or set of maximal cusps with disjoint interiors, then the length of a geodesic corresponding to an unknotting tunnel is less than $\ln (4)$.

For one-cusped manifolds, it is not generally known that an unknotting tunnel must be isotopic to a geodesic. However, in [2], it was shown to be true for hyperbolic 2-bridge knots. In [6], it was proved that an unknotting tunnel in a one-cusped hyperbolic manifold coming from 'generic' surgery on a 2-cusped manifold is isotopic to a geodesic. Moreover, in [7], it was proved that unknotting tunnels in one-cusped hyperbolic 3-manifolds can be arbitrarily long, unlike the case for 2-cusped manifolds. Explicit knot complements in $S^{3}$ with arbitrarily long unknotting tunnes were given in [6].

Given a vertical geodesic $\alpha$ in the manifold, we find sufficient conditions for it to be an unknotting tunnel. In particular, in the universal cover $H^{3}$, define an $n$-bracelet to be a cycle of $n$ horoballs covering the cusps, connected by lifts of $\alpha$. We show that if $\alpha$ has length less than $\ln (\sqrt{2})$ and possesses a 4-bracelet, it must be an unknotting tunnel. If $\alpha$ has length less than 0.16175 , and possesses a 5-bracelet, then it must be an unknotting tunnel. If $\alpha$ has length zero, meaning it corresponds to a point of tangency of the maximal cusp or cusps, it is an unknotting tunnel whenever there is a bracelet of six or fewer horoballs.

We then make use of the elder sibling property to obtain additional sufficient conditions for a geodesic in a knot complement to be an unknotting tunnel. The elder sibling property for balls in hyperbolic space was introduced by Freedman and McMullen in [8], where it was used to create a criterion for tameness of 3-manifolds. A 3-manifold is tame if it is homeomorphic to the interior of a compact manifold with boundary. In 1974 [9], Marden first raised the question of whether every complete hyperbolic 3-manifold with finitely generated fundamental group is topologically tame, and this question became known as the tameness conjecture. Marden proved that geometrically finite hyperbolic 3-manifolds are topologically tame. Freedman and McMullen developed the concept of elder sibling to make further inroads on the problem, proving with a Morse theory argument that the elder sibling property for a hyperbolic 3-manifold with finitely generated fundamental group implies tameness.

The tameness conjecture was eventually proved in full generality in 2004 by Agol [3], and by Calegari and Gabai [4]. A number of results follow from tameness, including the Ahlfors conjecture, posed in the early 1960s, which states that the limit set of a finitely generated Kleinian group is either the whole sphere, or of measure zero [5]. 
Freedman and McMullen define the elder sibling property to hold for a collection of open balls in $H^{3}$ if there exists a ball $B_{1}$ in the set such that any ball in the collection is joined to $B_{1}$ by a finite chain of overlapping balls moving monotonically closer to $B_{1}$.

We apply a version of the elder sibling property to collections of horoballs and beams connecting the horoballs corresponding to a cusped hyperbolic 3-manifold and geodesic pair, and show that if the ball-and-beam pattern satisfies the elder sibling property and has length less than $\ln 2$, the geodesic must be an unknotting tunnel.

Note that we are only considering orientable manifolds throughout, so any mention of manifolds should be taken to mean orientable manifolds, even when this is not specified.

\section{Ball-and-beam patterns}

To prove the following results, we make use of the ball-and-beam pattern associated with a given manifold and vertical geodesic pair $(M, \alpha)$, as discussed in [1]. A vertical geodesic is a geodesic $\alpha$ in a cusped hyperbolic 3-manifold $M$ that is perpendicular to the cusp or cusps at each of its ends. When the manifold is lifted to the upperhalf-space model of $H^{3}$ so that one end of a lift of the geodesic touches the horoball centered at $\infty$, the lift is a vertical ray.

The ball-and-beam pattern associated with $(M, \alpha)$ is the subset of $H^{3}$ given by $p^{-1}(C \cup N(\alpha))$, where $C$ is a cusp or the union of disjoint cusps. The beams are given by the preimage of $N(\alpha)$. We only consider a beam connected to a ball if its endpoint is at the center of the ball (which in the case of a horoball is the point of tangency of the ball with $\partial H^{3}$ ). It is possible that a beam may intersect a ball that is not one of the two balls at which it has its endpoints, but we consider these intersections to be ghost intersections, and they do not count as true intersections when we consider the connectedness of a ball-and-beam pattern. One can always shrink back the cusp or cusps so that the only intersections of beams with balls occurs when the balls are at the endpoints of the beams.

However, it is also convenient to be able to expand the balls. A maximal cusp of a manifold is a cusp that has been expanded until it first touches itself on the boundary. A maximal cusp then lifts to a set of horoballs in $H^{3}$, some of them tangent to each other, with disjoint interiors. In the case of multiple cusps, a maximal cusp collection is any choice of expanded cusps such that none overlap in their interiors, either with themselves or each other, and none can be expanded while preserving this fact. We define the length of a vertical geodesic to be the length in $H^{3}$ of that part of the geodesic that lies between the two points on the geodesic where it intersects the boundary of the maximal cusp or cusps (again excluding ghost intersections).

To distinguish between horoballs in a horoball pattern, we refer to a particular horoball as $H_{a}$, where $a$ is the point in the $x y$-plane at which the horoball is tangent. The horoball centered at $\infty$ will be denoted $H_{\infty}$. Most of the horoball patterns depicted in this paper correspond to maximal cusps.

It will also be useful to consider $n$-bracelets and $n$-disks in ball-and-beam patterns. An $n$-bracelet is a sequence of $n$ horoballs cyclically connected by beams. 


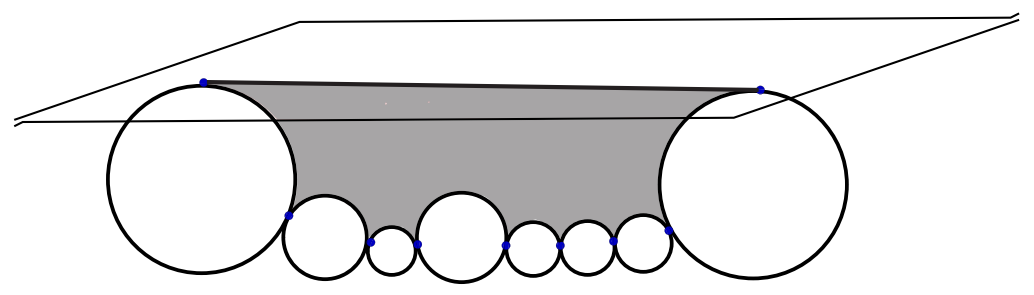

FIGURE 1. Here, $\alpha$ is a vertical geodesic of length zero, the lifts of which appear as dots corresponding to points of tangency of the horoballs. The nine horoballs form a 9-bracelet. The shaded region forms a 9-disk.

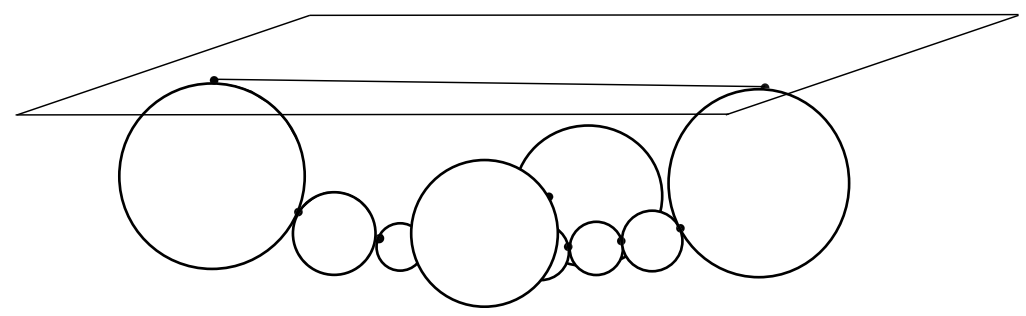

FIGURE 2. A blocked $n$-disk. A beam now intersects the obvious disk from Figure 1.

We assume $n \geq 3$. We say that a ball-and-beam pattern contains an $n$-disk if there is a disk $D$ in $H^{3}$ that intersects the ball-and-beam pattern of $(M, \alpha)$ in $\partial D$ such that $\partial D$ is a nontrivial curve in an $n$-bracelet. See Figure 1 for an example of how an $n$-bracelet might look. We say the $n$-bracelet is blocked if it does not correspond to an $n$-disk, that is, there is no nontrivial curve in the $n$-bracelet that bounds a disk with interior in the complement of the balls and beams (Figure 2).

In fact, a result from [1] renders these $n$-bracelets extremely useful for us.

Lemma 2.1 [1, Corollary 4.2]. If $M$ is a one-cusped hyperbolic 3-manifold and $\alpha$ a vertical geodesic within it or if $M$ is a 2-cusped hyperbolic 3-manifold and $\alpha$ is a vertical geodesic that has ends at both cusps, and if the ball-and-beam pattern for $(M, \alpha)$ contains an $n$-disk, then $\alpha$ must be an unknotting tunnel for $M$.

In the following section, we use $n$-disks and ball-and-beam patterns to provide sufficient conditions for a given vertical geodesic to be an unknotting tunnel. But first, we need a few geometric lemmas.

LEMMA 2.2. Let $H_{1}$ and $H_{2}$ be horoballs of Euclidean radii $r_{1}$ and $r_{2}$ centered at points $x_{1}$ and $x_{2}$ on the $x y$-plane, respectively, and let $\gamma$ be the geodesic that runs from $x_{1}$ to $x_{2}$. Let $b$ be the Euclidean radius of the semicircular arc formed by $\gamma$, and let the segment of $\gamma$ that runs between $H_{1}$ and $H_{2}$ have length $g$ (see Figure 3). Then $g=\ln \left(b^{2} /\left(r_{1} r_{2}\right)\right)$. 


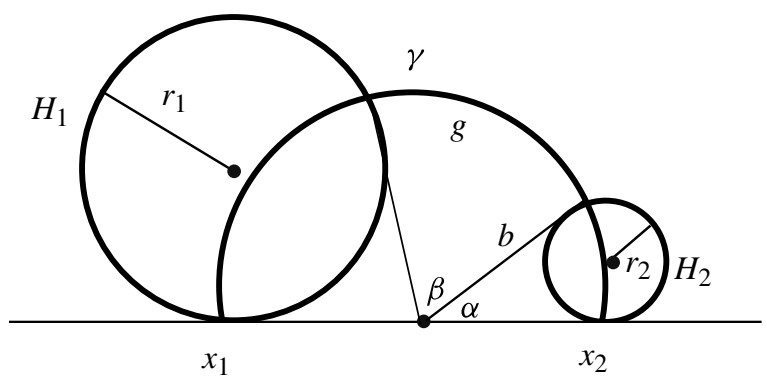

FIGURE 3. The situation described in Lemma 2.2.

Proof. The hyperbolic length of the geodesic arc connecting $H_{1}$ and $H_{2}$ is

$$
\ln \left(\frac{\csc \alpha-\cot \beta}{\csc \alpha-\cot \alpha}\right)=\ln \left(\frac{(1-\cos \beta)(\sin \alpha)}{(1-\cos \alpha)(\sin \beta)}\right),
$$

where $\alpha$ and $\beta$ are the angles shown in Figure 3. Calculating the point of intersection of $H_{1}$ and the semicircular arc of radius $b$, we can show that $\cos \alpha=\left(b^{2}-r_{1}^{2}\right) /\left(b^{2}+\right.$ $\left.r_{1}^{2}\right)$ and $\sin \alpha=\left(2 b r_{1}\right) /\left(b^{2}+r_{1}^{2}\right)$. Similarly, $\cos \beta=\left(b^{2}-r_{2}^{2}\right) /\left(b^{2}+r_{2}^{2}\right)$ and $\sin \alpha=$ $\left(2 b r_{2}\right) /\left(b^{2}+r_{2}^{2}\right)$. Substituting these values for $\sin \alpha, \cos \alpha, \sin \beta$, and $\cos \beta$ into the above formula for $g$ and simplifying yields that $g=\ln \left(b^{2} /\left(r_{1} r_{2}\right)\right)$.

LEMMA 2.3. For two horoballs $H_{1}$ and $H_{2}$ of radius $r_{1}$ and $r_{2}$ centered at points $x_{1}$ and $x_{2}$, respectively, on the $x y$-plane and connected by a geodesic arc segment of length $g$, the distance $d\left(x_{1}, x_{2}\right)$ between their points of tangency with the xy-plane is equal to $2 \sqrt{r_{1} r_{2} e^{g}}$.

Proof. The distance between the two horoballs is twice the Euclidean radius $b$ of the geodesic arc that runs from $H_{1}$ to $H_{2}$. By the lemma above, $g=\ln \left(b^{2} / r_{1} r_{2}\right)$, and so $d\left(x_{1}, x_{2}\right)=2 b=2 \sqrt{r_{1} r_{2} e^{g}}$.

Lemma 2.4. Let $x_{1}$ and $x_{2}$ be two points on the equator of a horosphere. Let $\alpha$ be the angle formed at the North Pole $N$ of the horosphere, between the arcs from $N$ to $x_{1}$ and $x_{2}$. Then the distance on the horosphere between $x_{1}$ and $x_{2}$ is given by $\sqrt{2-2 \cos \alpha}$.

Proof. The distance between $N$ and a point on the equator on any horosphere is exactly 1. The resultant triangle with vertices $x_{1}, x_{2}$ and $N$ is a Euclidean isosceles triangle with two edges of length 1 and the angle between them $\alpha$. Hence, the law of cosines yields the result.

Lemma 2.5. Given a bracelet consisting of balls with fixed centers and radii, the shortest possible beam that blocks it must either have length zero, or both of the blocking balls must be tangent to at least three of the bracelet balls.

Proof. Apply an isometry to center one of the blocking balls at $\infty$ so that the blocking beam becomes vertical. It clearly must be tangent to one of the bracelet balls or we 
could expand it to shorten the beam. The other blocking ball is centered in the plane. If it does not touch three of the bracelet balls, we can expand it, shrinking the length of the beam until the ball either touches three of the bracelet balls, or it touches the blocking ball at $H_{\infty}$, meaning the beam has length zero. So, assuming the beam does not have length zero, it must be that this blocking ball touches three of the bracelet balls. Now, placing this ball so it is centered at $\infty$ and repeating the argument implies that the other blocking ball must also touch three bracelet balls.

We now use these lemmas to provide conditions that ensure that a bracelet of length four, five or six in a ball-and-beam pattern for a manifold of one or two cusps corresponds to an $n$-disk. Note that we need not consider the case where a ball-andbeam pattern contains a 3-disk. By [1, Lemma 5.1], given a noncompact, orientable hyperbolic 3-manifold $M$ and vertical geodesic $\alpha$, the ball-and-beam pattern for $(M, \alpha)$ cannot contain a 3 -disk.

Proposition 2.6. Let $M$ be a hyperbolic 3-manifold of one or two cusps and let $\alpha$ be a vertical geodesic of length less than $\ln (\sqrt{2})$. If $M$ has two cusps, we require that $\alpha$ runs from one cusp to another. Then, if the ball-and-beam pattern of $(M, \alpha)$ contains a 4-bracelet, $\alpha$ must be an unknotting tunnel.

Proof. Suppose we have a hyperbolic manifold of one or two cusps with vertical geodesic $\alpha$ such that the hyperbolic length of $\alpha$ in $H^{3}$ is $g$. We will show that for small $g$, the wrist hole of a 4-bracelet becomes 'too small' to be blocked by other balls and beams.

Fix a particular choice of a 4-bracelet by fixing the centers and radii of the corresponding four horoballs, such that the lengths of the connecting geodesics are all identical, and equal to $g$. If any choice of a pair of horoball centers and radii and their corresponding beam could possibly block this bracelet, we take the minimal length blocking beam and corresponding pair of horoballs. Note that if $g=0$, there is no blocking beam of length zero possible. Hence we may assume that the length of the blocking beam is greater than zero. Since there are only four balls in the bracelet, Lemma 2.5 implies there is a bracelet ball that is tangent to both of the blocking balls.

We apply an isometry taking that bracelet ball to $H_{\infty}$, which we take to have Euclidean height one. For convenience, we label the two horoballs in the 4-bracelet connected to $H_{\infty}$ as $H_{a}$ and $H_{b}$, and the ball in between them $H_{c}$. Note that since $H_{a}$ and $H_{b}$ are both connected to $H_{\infty}$ by a segment of $\alpha$ with length $g$, they are the same distance from $H_{\infty}$ and thus have the same Euclidean height. Let the two blocking balls be labelled $H_{e}$ and $H_{f}$. Since both are tangent to $H_{\infty}$, they both have diameter one.

Hence, to obtain the shortest possible blocking beam, we want them to have the shortest possible Euclidean distance between their centers on the $x y$-plane. However, this occurs when both are tangent to $H_{a}$ and $H_{b}$, and the centers of $H_{a}$ and $H_{b}$ are as far apart as possible. This happens when $c$ is in line with $a$ and $b$, as in Figure 4. However, then we can apply an isometry that takes the geodesic line on the $x y$-plane through the centers of the bracelet balls and $\infty$ to the circle of radius $\sqrt{2}$ in the complex 


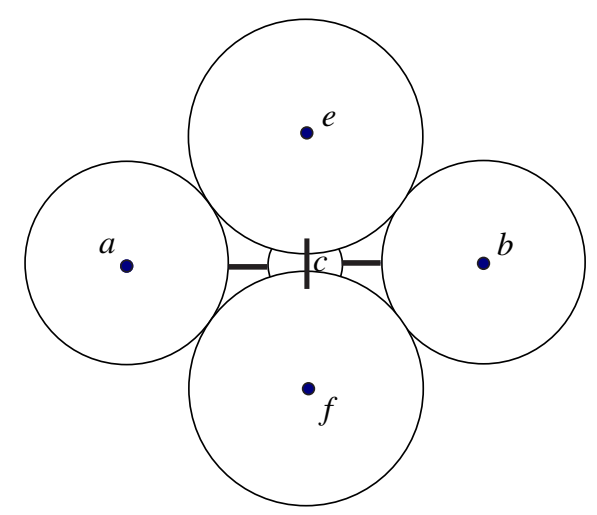

Figure 4. A view from above of a blocked 4-disk.

plane so that the four centers of the bracelet balls occur at $\pm 1 / 2 \pm i / 2$. Then the two blocking balls must be centered at $\infty$ and 0 . Begin with the four bracelet balls having radius $1 / 2$. Then they touch each other and the length of the beam connecting them sequentially is zero. The shortest beam that could block the 4-disk with boundary in the bracelet corresponds to the blocking balls $H_{\infty}$, with boundary the horizontal plane at height one, and $H_{0}$, which is the horoball centered at the origin, tangent to all four balls in the bracelet, and hence of radius $1 / 4$. Note that the vertical beam connecting $H_{\infty}$ and $H_{0}$ then has length $\ln 2$. To have an actual case of a beam blocking a disk, it must be that the beams in the bracelet have the same length as the blocking beam. So we will shrink back the size of the horoballs in the bracelet and expand the size of the blocking balls until the two beam lengths match.

Suppose the radii of the balls in the bracelet are $a$. Let $g_{1}$ be the length of the beam in the bracelet, a function of $a$. Let $g_{2}$ be the length of the beam connecting the corresponding $H_{\infty}$ and $H_{0}$. Then the horizontal plane that bounds $H_{\infty}$ is at height $2 a$. Let $r$ be the radius of the horoball $H_{0}$. The center of $H_{0}$ is a distance $1 / \sqrt{2}$ from the center of each of the balls in the bracelet. Considering one of them, it must then be the case that $(1 / \sqrt{2})^{2}+(a-r)^{2}=(a+r)^{2}$. Thus, $r=1 /(8 a)$, and $g_{2}=\ln (2 a / 2 r)=\ln \left(8 a^{2}\right)$.

Lemma 2.2 implies that $g_{1}=\ln \left((1 / 2)^{2} / a^{2}\right)$. Setting $g_{1}=g_{2}$, we obtain $a=1 /(2 \sqrt[4]{2})$, and hence $g_{1}=g_{2}=\ln (\sqrt{2})$.

Hence, for a 4-bracelet to be blocked, $g$ must be at least $\ln (\sqrt{2})$. So if $g<\ln (\sqrt{2})$ and if the ball-and-beam pattern for $(M, \alpha)$ contains a 4-bracelet, then the 4-bracelet bounds a disk, and so $\alpha$ must be an unknotting tunnel.

Proposition 2.7. Let $M$ be a one-cusped hyperbolic 3-manifold and let $\alpha$ be a vertical geodesic of length less than $\ln (2 \cos (3 \pi / 10)) \approx 0.16175$. Then, if the ball-and-beam pattern associated with $(M, \alpha)$ contains a 4-bracelet or a 5-bracelet, $\alpha$ must be an unknotting tunnel. 


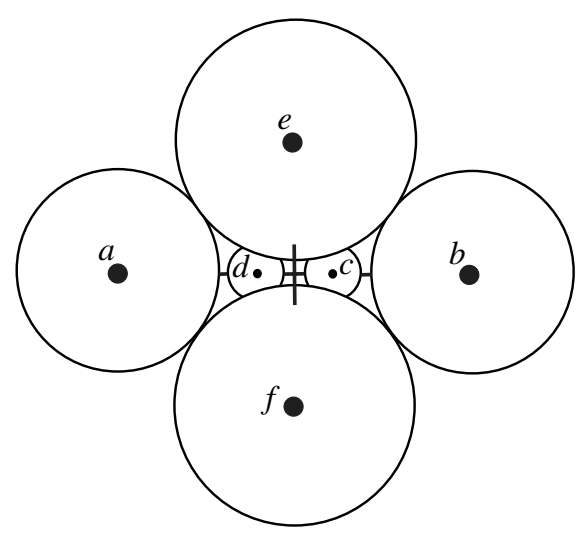

FIGURE 5. A view from above of a blocked 5-bracelet showing the beams corresponding to $\alpha$.

Proof. If the ball-and-beam pattern for $(M, \alpha)$ contains a 4-bracelet, then the fact that $\alpha$ is an unknotting tunnel follows from Proposition 2.6. All that remains to show is that for sufficiently small vertical geodesic length $g$, it is impossible for a 5-bracelet to be blocked.

We will show that among all 5-bracelets corresponding to a given length $g$, the 5-bracelet where the two blocking balls are tangent to all five of the bracelet balls yields the minimal length for a blocking beam. Choose any particular conformation for the 5-bracelet, fixing the centers and radii of the bracelet balls. Let $H_{e}$ and $H_{f}$ be the blocking balls corresponding to a blocking beam of least possible length for that conformation. Lemma 2.5 states that if the beam has length greater than zero, $H_{e}$ and $H_{f}$ are each tangent to three of the bracelet balls, and therefore they are tangent to a shared bracelet ball. Again,we apply an isometry to all the balls that takes this bracelet ball to $H_{\infty}$ at height one. The other horoballs of the resulting 5-bracelet are labelled $H_{a}, H_{b}, H_{c}$ and $H_{d}$ as in Figure 5. As before, $H_{a}$ and $H_{b}$ are both a hyperbolic distance $g$ from $H_{\infty}$, and so have the same radius. We have that $H_{e}$ and $H_{f}$ have diameter one, since they are tangent to $H_{\infty}$. The case that will minimize the length of the geodesic from $H_{e}$ to $H_{f}$ is when $H_{e}$ and $H_{f}$ are as close together as possible, hence when both are tangent to each of $H_{a}$ and $H_{b}$ and $H_{a}$ and $H_{b}$ are as far apart as possible. This occurs when $c$ and $d$ are in line with $a$ and $b$.

Since $H_{e}$ and $H_{f}$ are both tangent to $H_{a}$, we can apply an isometry that takes $H_{a}$ to $H_{\infty}$. By the same argument, the images of $H_{e}$ and $H_{f}$ must be tangent to the image of $H_{d}$. Doing the same for $H_{b}$ implies that in fact $H_{e}$ and $H_{f}$ must be tangent to all five of the horoballs in the bracelet.

Applying an isometry that takes $H_{e}$ to $H_{\infty}$, and $H_{f}$ to $H_{0}$, we see that the centers of the bracelet horoballs must form the vertices of a regular pentagon with center at the origin. Choosing the length of the edges of the regular pentagon to be one, the vertices of the pentagon are all a distance $1 /(2 \cos (3 \pi / 10))$ from the origin. 


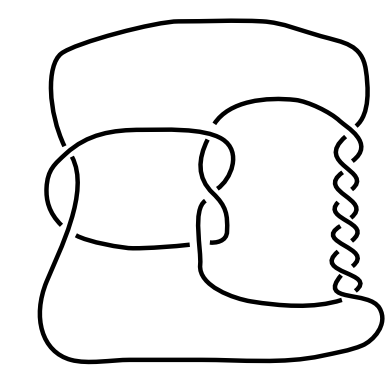

Figure 6 . The $(-2,3,7)$ pretzel knot.

Suppose the radii of the balls in the bracelet are all $a$. Let $g_{1}$ be the length of the beam in the bracelet, which by Lemma 2.2 is given by $g_{1}=\ln \left((1 / 2)^{2} / a^{2}\right)$. Let $r$ be the radius of the horoball $H_{0}$ and let $g_{2}$ be the length of the beam connecting the corresponding $H_{\infty}$ and $H_{0}$. Since the horizontal plane that bounds $H_{\infty}$ is at height $2 a, g_{2}=\ln (2 a / 2 r)$. The center of $H_{0}$ is a distance $1 /(2 \cos (3 \pi / 10))$ from the center of each of the balls in the bracelet. Considering one of them, it must then be the case that $(1 /(2 \cos (3 \pi / 10)))^{2}+(a-r)^{2}=(a+r)^{2}$. Thus, $r=1 /\left(16 a \cos ^{2}(3 \pi / 10)\right)$, and $g_{2}=\ln \left(16 a^{2} \cos ^{2}(3 \pi / 10)\right)$.

Setting $g_{1}=g_{2}$, we obtain $a=1 /(2 \sqrt{2} \sqrt{\cos (3 \pi / 10)})$, and hence $g_{1}=g_{2}=$ $\ln (2 \cos (3 \pi / 10))$.

Thus, if $g<\ln (2 \cos (3 \pi / 10))$, a 5-bracelet cannot be blocked, and so in this case, the presence of a 5-bracelet implies that the ball-and-beam pattern of the manifold and geodesic pair $(M, \alpha)$ contains an $n$-disk and hence $\alpha$ is an unknotting tunnel.

Although it may seem that perhaps relatively few vertical geodesics have length $g<0.16175 \ldots$, note that in fact, in every one-cusped manifold there is a vertical geodesic of length zero. The maximal cusp is obtained by expanding the cusp until it first becomes tangent to itself, and the arc that passes perpendicularly through this point of tangency with endpoints perpendicular to the two cusps is a vertical geodesic of length zero. Moreover, 5-bracelets do arise in ball-and-beam patterns for orientable manifolds, as in this example of the $(-2,3,7)$-pretzel knot (see Figure 6), which has a horoball pattern as appears in Figure 7 where we take the vertical geodesic of length zero corresponding to tangency points of the horoballs.

We conclude by noting that the same type of argument does not work to find a value for $g$ below which an $n$-bracelet in the ball-and-beam pattern must correspond to an $n$-disk, for $n$ equal to 6 or greater. Consider Figure 8 where we see an arrangement of horoballs and beams corresponding to a geodesic of length zero, where a 6-bracelet defined by the hexagon is blocked by the geodesic with endpoints at the center horoball and the horoball at infinity and thus does not correspond to an $n$-disk.

However, one can show that in fact, the configuration depicted cannot occur for an orientable hyperbolic 3-manifold of one or two cusps. 


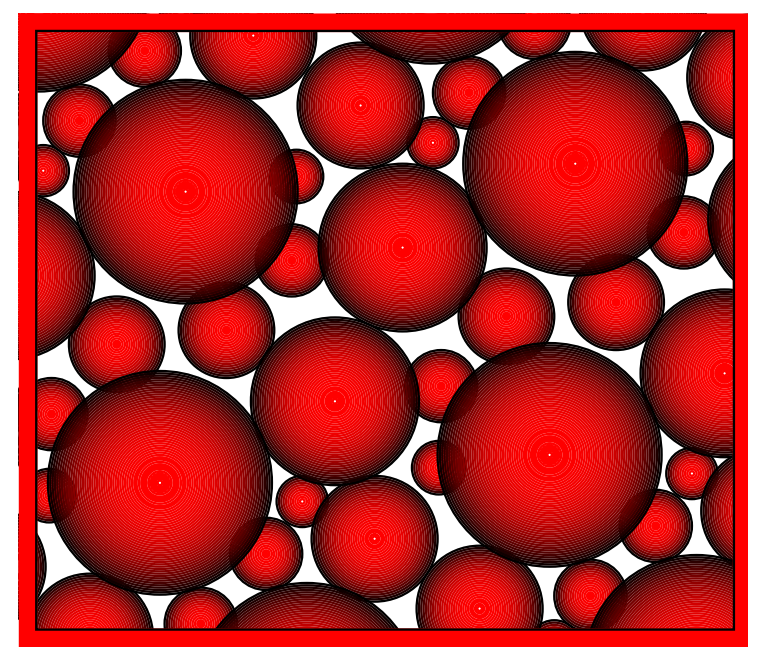

FIGURE 7. A section of the horoball pattern for the $(-2,3,7)$ pretzel knot produced by [11]. When the proper length zero vertical geodesic is added to make this a ball-and-beam pattern, the sequence of the four tangent horoballs running from the top right to the bottom left in this illustration, together with $H_{\infty}$ form an unblocked 5-bracelet that corresponds to a 5-disk.

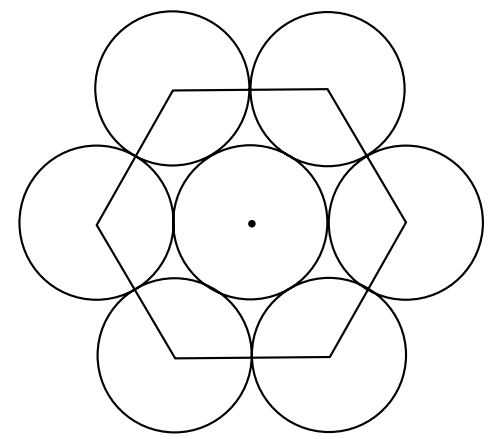

FIGURE 8. A blocked six-bracelet when $g=0$.

Proposition 2.8. Let $M$ be a hyperbolic 3-manifold of one or two cusps and let $\alpha$ be a vertical geodesic of length zero. If there are two distinct cusps, $\alpha$ connects them. Then, if the ball-and-beam pattern associated with $(M, \alpha)$ contains a bracelet of length four, five, or six, then $\alpha$ must be an unknotting tunnel.

Proof. By Propositions 2.6 and 2.7, we need only consider bracelets of length six. We show that the local situation that must occur for a bracelet of length six to be blocked when $\alpha$ has length zero cannot occur.

Suppose a bracelet of length six is blocked. Choosing one of the blocking horoballs to be the horoball at infinity, we obtain a picture as in Figure 8, where each of the six depicted edges and the vertical edge at the center are all in the same edge class. 


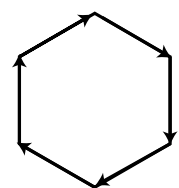

(1)

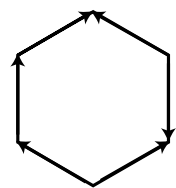

(4)

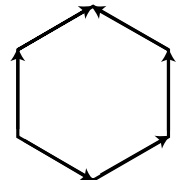

(7)

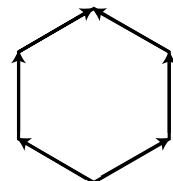

(2)

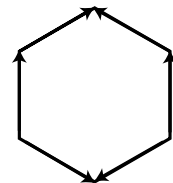

(5)

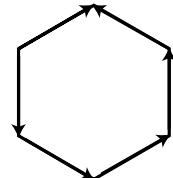

(8)

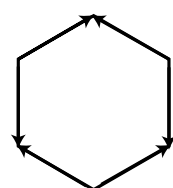

(3)

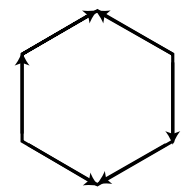

(6)

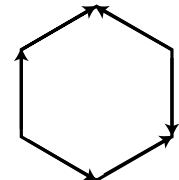

(9)

FIGURE 9. Possible orientations on the 6-bracelet edges.

Orienting all of these edges consistent with their identifications, we assume the vertical edge at the center points up out of the $x y$-plane. Considering the orientations on the edges in the bracelet, one finds that up to rotation and reflection, there are nine possibilities, listed in Figure 9.

We normalize so that the diameter of the horoballs depicted is one and call the hexagon $Q$. In general, we seek a contradiction by showing that there is an isometry in the fundamental group that fixes points.

We note several facts. First, any time an edge is identified to another edge of the same equivalence class but with opposite orientations, the midpoint is fixed by the identification, yielding a contradiction.

Second, in the case of two cusps, the oriented arrows must always have their tails in horoballs corresponding to the first cusp and their heads in horoballs corresponding to the second cusp. Hence, no horoball can contain both heads and tails, and the only diagram in Figure 9 that can occur is case (3).

Third, in the case of one cusp, there must be an isometry that takes $H_{0}$ to $H_{\infty}$. This will send the hexagon $Q$ of six horoballs to another hexagon in the cusp diagram, however, with the hexagon and its arrow labels flipped, so that the arrow orientations read counterclockwise as they did before clockwise. At its center is an arrow that points down. We call this a flipped hexagon.

In the case of a diagram with the tail of an arrow meeting the head of the next arrow, as occurs for all but case (3), Lemma 2.4 implies that there must be a downward pointing vertical arrow a Euclidean distance exactly $\sqrt{3}$ in the $x y$-plane from the 
upward pointing vertical arrow at the center of the original hexagon. This downward pointing arrow will be at the center of a flipped hexagon, which, because horoballs cannot overlap in their interiors, will overlap with the original hexagon on exactly one edge of their boundaries.

For any of the cases of hexagons that have opposite edges with parallel orientations, which includes all but cases (1) and (6), there must be a corresponding parabolic translation fixing $\infty$ that identifies the two edges, and hence slides points a Euclidean distance $\sqrt{3}$. This translation and its inverse have the effect of gluing additional unflipped hexagons to either side of the first along each of the opposite edges with parallel orientations.

We now consider cases. For case (1), all of the edges on the flipped hexagon have opposite orientation from the edges on the original hexagon, and hence the flipped and unflipped hexagons cannot share an edge, a contradiction. For case (2), there are three pairs of opposite edges that are parallel. Hence the original hexagon is surrounded by unflipped hexagons and there is no room for a flipped hexagon, a contradiction. For case (3), it cannot correspond to one cusp, for the same reason as case (2). There are three pairs of opposite edges of parallel orientation, meaning there is no room for the flipped copy. We will return to the possibility of two cusps for case (3) at the end of the proof.

In each of the cases (4) and (7), there are two pairs of opposite edges with parallel orientations. This generates two parabolic isometries $f$ and $g$. We can choose $f$ and $g$ so that $f(Q)$ and $g(Q)$ share an edge. However, in both of these cases, that edge of the images has opposite orientations, a contradiction.

For cases (5) and (9), there is a single pair of opposite edges with parallel orientations. This generates a translation $f$ by $\sqrt{3}$, and by applying $f$ and its inverse to $Q$, we fill two slots where hexagons could go around $Q$. There remain four slots where a flipped hexagon could go, but in all cases, although the orientations of the edges of the flipped version could match the orientations of the edges of $Q$ and its images that it would be glued to, the orientations of the rest of the edges of the flipped version would not respect $f$ in the sense that $f$ would identify two edges with opposite orientations, a contradiction.

Case (6) has two arrowheads meeting at a horoball with an angle of $120^{\circ}$. Hence, by Lemma 2.4 , there must be a second vertical upward pointing edge a distance $\sqrt{3}$ from the original central edge of $Q$. Since these two upward pointing edges must be identified by a parabolic translation fixing $\infty$, there must be two opposite edges on $Q$ with parallel orientations, a contradiction to this case.

To eliminate cases (8) and (3) for two cusps, we will also consider the fact that each hexagon is the projection of a region in hyperbolic space that can be subdivided into six vertical ideal regular tetrahedra, the edges of which all pass through points of tangency of the eight horoballs depicted (including $H_{\infty}$ ). The single labelled edge class includes 12 of the edges of these tetrahedra, six around the outside of the hexagon and six meeting along the central edge, which together subtend an angle of $720^{\circ}$. Hence some of these six tetrahedra must be identified with one another, and they can generate at most three tetrahedral equivalence classes. 


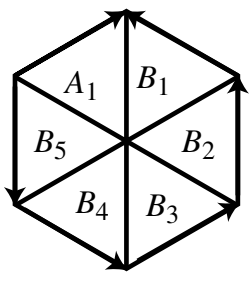

(8)

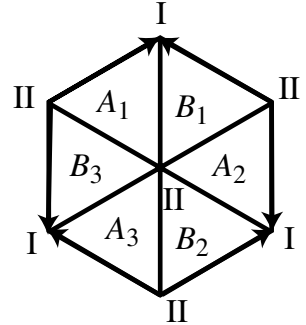

(3)

Figure 10. Labelling tetrahedra for cases (8) and (3).

Note that no two tetrahedra can be identified by an identification that sends the central edge of one to the central edge of another, as then that edge would be a fixed point set for the isometry, contradicting the fact that all isometries in the fundamental group must be fixed point free. Define a standard identification to be when tetrahedron $T_{1}$ is identified to $T_{2}$ with an orientation preserving isometry such that the central edge and outer edge of $T_{1}$ go to the outer edge and central edge of $T_{2}$, respectively.

Suppose that there is a nonstandard identification between two tetrahedra $T_{1}$ and $T_{2}$. Then a labelled edge on $T_{1}$ must go to an unlabelled edge on $T_{2}$. Since the labelled edges are opposite pairs, both of the labelled edges on $T_{1}$ must go to unlabelled edges. Hence, there are four edges in this equivalence class on each of the two tetrahedra. Since, after tetrahedral identification, there can be at most six such edges in the edge class, this implies that there can be only two classes of tetrahedra, one with all tetrahedra having four edges in the edge class, excluding an opposite pair of edges, and one with all tetrahedra having two edges in the edge class that are an opposite pair. Call a representative of each tetrahedral class $A$ and $B$, respectively. Then all faces of $A$ have two edges in the equivalence class, whereas no face of $B$ has two edges in the equivalence class. Hence, there is no way to glue faces of $A$ to faces of $\mathrm{B}$ without adding edges to the edge class, a contradiction to the fact there are at most six edges in the edge class after identifications. Hence this cannot occur, and all identifications of tetrahedra must be standard.

If two of the tetrahedra are identified, their outer edges must have the same orientation, either clockwise or counterclockwise on the hexagon, since the identification of $T_{1}$ with $T_{2}$ must send the outer edge of $T_{1}$ to the central edge of $T_{2}$ and the central edge of $T_{1}$ to the outer edge of $T_{2}$.

For case (8), we know that the two opposite edges with parallel orientations are identified by a parabolic isometry $p$ fixing $\infty$. Label the tetrahedra as in Figure 10. Since one outer edge is oriented one way, while the other five are oriented the other, tetrahedron $A_{1}$ cannot be identified to any others. Since the parabolic isometry puts a copy of $A_{1}$ next to $B_{3}$, and no other tetrahedron can be identified to $A_{1}$, the only tetrahedron that could be identified to $B_{3}$ is $B_{1}$, as the standard identification $f$ that 
does so would also send a copy of $A_{1}$ to $p\left(A_{1}\right)$. However, then $p^{-1} f\left(A_{1}\right)=A_{1}$ and $p^{-1} f$ is a nontrivial element of the group of isometries with a fixed point, a contradiction.

So both $A_{1}$ and $B_{3}$ must be their own equivalence classes, which implies that the other four tetrahedra are identified with one another. But if $B_{1}$ is identified to $B_{2}$ by the standard identification $g$, then $g\left(A_{1}\right)$ will be adjacent to $B_{2}$. If $B_{5}$ is identified to $B_{2}$ by $h$, then $h\left(B_{4}\right)$ is adjacent to $B_{2}$ and is therefore identified with $g\left(A_{1}\right)$, contradicting the fact that $A_{1}$ is the only tetrahedron in its equivalence class. So this case cannot occur.

Our last case is (3) when it has two cusps. We label the horoballs either with a I or a II, depending on the cusp each corresponds to. Note that the horoball at $\infty$ corresponds to cusp I. Then we label the tetrahedra into an $A$ class and a $B$ class, each of which has three tetrahedra, depending on the orientation of their outer edge, as in Figure 10.

We consider the three vertical edges $v_{1}, v_{2}$ and $v_{3}$ that go from horoballs labelled I to $H_{\infty}$ and that are on $A_{1}, A_{2}$ and $A_{3}$, respectively. Each corresponds to an edge that goes from cusp I back to cusp I. If $A_{1}$ is identified to $A_{2}$, then $v_{1}$, which we orient up, must go to $v_{2}$ by the standard identification, which means it points down. Then $A_{3}$ cannot be identified to either $A_{1}$ or $A_{2}$ since $v_{3}$ must either point up or down, and the identifications with each of these tetrahedra would force opposite orientations on $v_{3}$. Hence there are at least two equivalence classes of $A$ tetrahedra. The same argument shows that there are two equivalence classes of $B$ tetrahedra, implying there are at least four equivalence classes of these six tetrahedra, a contradiction.

Note that a vertical geodesic $\alpha$ of length zero can have a blocked 6-cycle of exactly the type described above if we allow the manifold to be nonorientable. See the manifold m025 from the cusped census of SNAPPEA [11], which consists of exactly three regular tetrahedra.

\section{The elder sibling property}

Next, we present the elder sibling property for a ball-and-beam pattern, which provides additional sufficient conditions for a vertical geodesic to be an unknotting tunnel.

DefinITION 3.1. A ball-and-beam pattern is said to satisfy the elder sibling property if every horoball $H_{a}$ in the pattern is connected to $H_{\infty}$ by an alternating sequence of horoballs and beams such that all of the balls in the chain have Euclidean radius greater than $H_{a}$. Such a chain of balls and beams will be called an elder sibling chain of $H_{a}$.

To better understand the properties of an elder sibling ball-and-beam pattern, we note the following.

LEMMA 3.2 [1]. In a ball-and-beam pattern, there are only finitely many Euclidean sizes of horoballs greater than or equal to a given value.

PRoof. The fundamental domain for the cusp is formed by a parallelogram on the $x y$ plane of finite area. But there is no room inside it for infinitely many balls tangent 
to the $x y$-plane of size greater than or equal to a given value. So there can be only finitely many horoballs of Euclidean radius greater than or equal to a given value in the fundamental domain, and hence there are only finitely many sizes of horoballs in the ball-and-beam pattern larger than a given size.

Notice that our definition of the elder sibling property is equivalent to stating that every horoball must be connected to $H_{\infty}$ by a sequence of balls and beams such that the Euclidean radius of the balls in the sequence is strictly increasing. This follows since if we start with a ball $H_{a}$, it must be connected to a ball of larger radius. That ball, in turn, is also connected to a ball of larger radius. Continuing in this manner and noting that Lemma 3.2 shows that there are only finiltely many sizes of balls above a given radius, we know these chains of horoballs of increasing size must be finite, ending at $H_{\infty}$.

Also, note that if a ball-and-beam pattern satisfies the elder sibling property, it must be connected, since the fact that every ball can be joined to $H_{\infty}$ implies that any two balls can be joined by a path that goes from one ball to $H_{\infty}$ and then from $H_{\infty}$ to the other ball. This fact will become useful with the help of an observation from [1].

Lemma 3.3 [1]. If a ball-and-beam-pattern is connected, it must contain an n-bracelet.

To formulate the next lemma, we suppose that an $n$-bracelet is blocked. Define a pair of blocking balls to be any pair of horoballs in the ball-and-beam pattern connected by a beam that punctures a disk with boundary a nontrivial curve in the $n$-bracelet.

Intuitively, it would seem that in the ball-and-beam pattern for a manifold and vertical geodesic pair $(M, \alpha)$, in order that an $n$-bracelet is blocked, the blocking balls need to be larger than a certain size in order to reach over the balls of the blocked $n$-bracelet. In fact, this is the case, and the size of these blocking horoballs will be related to the length of $\alpha$ and the size of the smallest ball of the blocked bracelet in the following way.

Lemma 3.4. Let $(M, \alpha)$ be a hyperbolic manifold and vertical geodesic pair, where $M$ has one or two cusps and if it has two cusps, $\alpha$ connects them. Let $\alpha$ have length $g<\ln 2$. Then if there exists an $n$-bracelet in the ball-and-beam pattern and it is blocked, there is a blocking ball in any blocking ball pair of radius at least $\left(2+\sqrt{4-e^{2 g}}\right) / e^{g}$ times the Euclidean radius of the smallest ball in the $n$-bracelet.

Proof. Suppose we have a blocked $n$-bracelet, with the smallest ball in the bracelet having Euclidean radius $r$. The beam connecting any pair of blocking balls must reach a sufficient vertical height to pass over the $n$-bracelet. Let $H_{e}$ and $H_{f}$ be a pair of blocking balls for the bracelet. We can assume that they are the same size since if the first were larger than the second, expanding the second while preserving $g$ only increases the vertical height attained by the beam. Let $D$ be the Euclidean distance between their centers and $R$ their radius. Then the line segment between their centers must intersect one of the line segment projections of the beams from the $n$-bracelet. 
We can assume that it intersects the projection of one of the beams leaving the smallest ball in the $n$-bracelet, since otherwise, the radius of $H_{e}$ and $H_{f}$ would need to be even larger. Let $H_{a}$ be the smallest ball and $H_{b}$ the ball connected to it by this beam. We can further assume that $H_{b}$ has the same radius $r$ as $H_{a}$. Let $d$ be the Euclidean distance between their centers. By Lemma 2.2, $g=\ln \left(d^{2} / 4 r^{2}\right)=\ln \left(D^{2} / 4 R^{2}\right)$.

Since balls cannot overlap, note that the centers of $H_{e}$ and $H_{f}$ must be a distance of at least $2 \sqrt{R r}$ from the centers of both $H_{a}$ and $H_{b}$. To minimize $R$, assume that $H_{e}$ is closer to $H_{a}$ than to $H_{b}$. We can then assume that $H_{e}$ and $H_{f}$ are both tangent to $H_{a}$ and by the Pythagorean theorem, $4 R r \leq d^{2} / 4+D^{2} / 4=r^{2} e^{g}+R^{2} e^{g}$. Hence, $R^{2}-4 R r / e^{g}+r^{2} \geq 0$. The quadratic formula then yields the result.

We are now ready to apply the elder sibling property as a criterion for a vertical geodesic to be an unknotting tunnel.

THEOREM 3.5. Let $M$ be a hyperbolic 3-manifold of one or two cusps with a vertical geodesic $\alpha$ that connects the cusps if there are two. If the ball-and-beam pattern for $(M, \alpha)$ satisfies the elder sibling property, and $\alpha$ has length less than $\ln (2)$, then $\alpha$ is an unknotting tunnel.

Proof. The elder sibling property implies that the ball-and-beam pattern for $(M, \alpha)$ is connected, and thus, by Lemma 3.3 it must contain an $n$-bracelet. We will show that the ball-and-beam pattern also contains an $n$-disk, which is enough to prove that $\alpha$ is an unknotting tunnel.

Since the ball-and-beam pattern for $(M, \alpha)$ contains an $n$-bracelet, we can choose an $n$-bracelet containing $H_{\infty}$, and let $\beta$ be the curve in this $n$-bracelet that passes through the sequence of $n$ balls cyclically connected by beams in order and connects back to itself. Let $H_{\min }$ be the smallest horoball in this $n$-bracelet. Clearly, $\beta$ is a nontrivial curve in the $n$-bracelet, so if it bounds a disk with interior in the complement of the ball-and-beam pattern, the ball-and-beam pattern allows an $n$-disk and hence $\alpha$ is an unknotting tunnel.

If, on the other hand, $\beta$ does not bound a disk, it must be the case that the obvious disk in $H^{3}$ bounded by $\beta$ is blocked in at least one place by other balls and beams, as shown in Figure 2.

Note that $\beta$ has finite length in $H^{3}$, and thus is contained in a finite volume of $H^{3}$, so there can only be finitely many such places where the disk is blocked. We can number them $1,2, \ldots, m$. At each place where the disk is blocked, we must have $\beta$ passing through a blocking disk, bounded by two arcs on horoballs and the beam connecting them, and an arc in the $x y$-plane, as shown in Figure 11 . We call the beam that connects the two blocking balls and runs along the boundary of the blocking disk the blocking beam.

Notice that if the disk corresponding to $\beta$ is not blocked by a pair of horoballs connected by a beam, but instead just by a horoball that intersects the disk, we can deform the disk bounded by $\beta$ to pass around the blocking ball to eliminate the block. At the first blockage, we choose the larger of the two horoballs whose connecting beam 


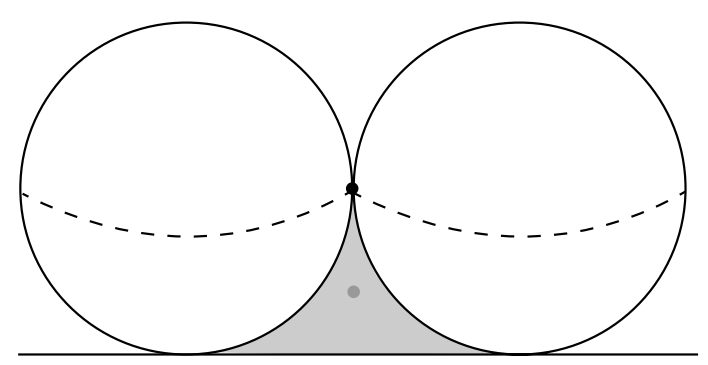

FIGURE 11. A view of one place where the $n$-bracelet bounded by $\beta$ is blocked. Here $\beta$ appears in cross section as a grey dot, the blocking beam as a black dot (assuming here the length of $\alpha$ is zero), and the blocking balls in grey. The blocking disk is shaded.

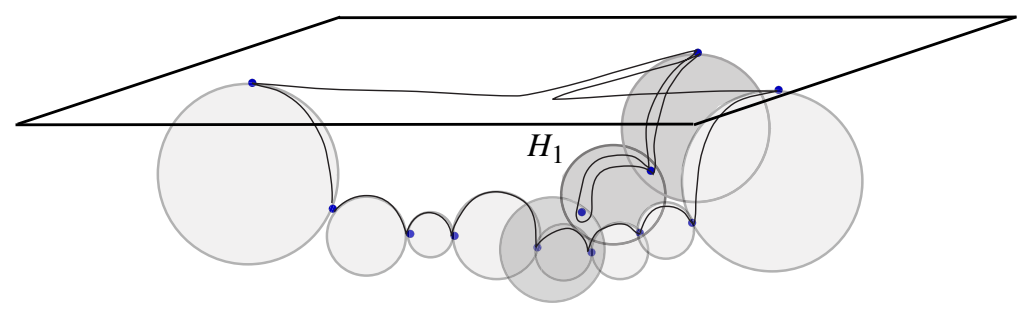

FIGURE 12. Modifying $\beta$ to eliminate a block.

is blocking the disk and call it $H_{1}$. For the bracelet to be blocked, $H_{1}$ must be at least $\left(2+\sqrt{4-e^{2 g}}\right) / e^{g}$ times the size of the balls that it is reaching over, and hence it must have Euclidean height of at least $\left(2+\sqrt{4-e^{2 g}}\right) / e^{g}$ times the Euclidean height of $H_{\text {min }}$. Since the length of $\alpha=g<\ln 2$, the quantity $\left(2+\sqrt{4-e^{2 g}}\right) / e^{g}$ is greater than one, and so this blocking ball is larger than $H_{\min }$. Furthermore, we can get from $H_{1}$ to $H_{\infty}$ via a chain of horoballs connected to one another by beams such that the Euclidean height of each ball in this chain is larger than the Euclidean height of the preceding ball, and so also larger than the Euclidean height of $H_{\min }$.

We will prove that we can obtain an $n$-bracelet with fewer blockages and smallest ball no smaller than the size of $H_{\text {min }}$. Repeating the process a finite number of times will then yield the disk that we seek.

We modify $\beta$ in the following manner. Take the ball $H_{1}$ and find an elder sibling chain of balls from it to $H_{\infty}$. We take the segment of $\beta$ that runs along $H_{\infty}$, choose a point $x$ on that segment, cut $\beta$ open along that point and then add in the following path. It runs from that point $x$ over to the top of the chain on $H_{\infty}$, down the chain to $H_{1}$, and while still on $H_{1}$, around the underside of the beam that blocked our original $n$-bracelet, then back up the elder sibling chain from $H_{1}$ to $H_{\infty}$, then back on $H_{\infty}$ to the starting point. For an example of how this process might look for a length zero vertical geodesic, see Figures 12 and 13.

We have created a new $n$-bracelet that is no longer blocked by our first blockage. Let the new curve created by modifying $\beta$ in this way be called $\beta_{2}$, and note that $\beta_{2}$ 


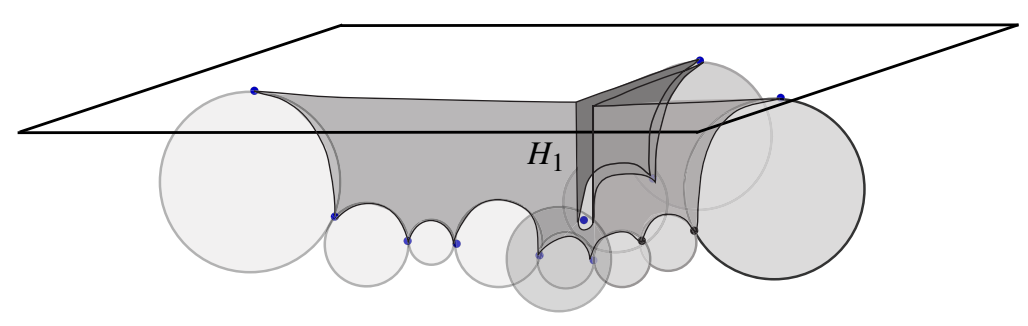

FIGURE 13. The disk bounded by the modified curve $\beta_{2}$.

is still a nontrivial curve in an $n$-bracelet. If $\beta_{2}$ has more blockages than did $\beta_{1}$, then these additional blocking balls incident to these blocking disks reach over the new elder sibling chain that $\beta_{2}$-but not $\beta$-runs along. These blocking horoballs correspond to new elder sibling chains extending up to $H_{\infty}$, each of whose smallest ball must be strictly larger than the smallest ball in the part of the bracelet that they are reaching over. Keep in mind there are only a finite number of sizes of balls bigger than $H_{\text {min }}$. Again, we repeat the process of modifying $\beta_{2}$, taking a segment of $\beta_{2}$ that runs along $H_{\infty}$, and running it down the elder sibling chain corresponding to each blockage, around $\alpha$, and back up to $H_{\infty}$, to create a new curve, which we call $\beta_{3}$.

It can be shown that this process cannot be continued indefinitely. At each successive modification of $\beta$, we involve elder sibling chains whose smallest horoball is strictly larger than the smallest horoball in the elder sibling chains obtained in the previous step. Thus, we obtain a sequence of horoballs of increasing size, all of which are larger than $H_{\min }$. Since there can be only finitely many sizes of horoballs larger than a given size in the cusp diagram, this sequence must be finite. Thus, our process of modifying $\beta$ must end within a finite number of steps, and so $\beta_{t}$ will correspond to an $n$-bracelet with fewer blockages. Repeating this process a finite number of times will produce an unblocked $n$-disk in the ball-and-beam pattern for $(M, \alpha)$, and it follows that $\alpha$ is an unknotting tunnel.

\section{References}

[1] C. Adams, 'Unknotting tunnels in hyperbolic 3-manifolds', Math. Ann. 302 (1995), 177-195.

[2] C. Adams and A. Reid, 'Unknotting tunnels in two-bridge knot and link complements', Comment. Math. Helv. 71(4) (1996), 617-627.

[3] I. Agol, 'Tameness of hyperbolic 3-manifolds' (2004), arXiv:math.GT/0405568.

[4] D. Calegari and D. Gabai, 'Shrinkwrapping and the taming of hyperbolic 3-manifolds', J. Amer. Math. Soc. 19(2) (2006), 385-446.

[5] R. Canary, 'Marden's tameness conjecture: history and applications', in: Geometry, Analysis, and Topology of Discrete Groups, Advanced Lectures in Mathematics (ALM), 6 (2008), 137-162.

[6] D. Cooper, D. Futer and J. Purcell, 'Dehn filling and the geometry of unknotting tunnels' (2011), arXiv:1105.3461.

[7] D. Cooper, M. Lackenby and J. Purcell, 'The length of unknotting tunnels', Algebr. Geom. Topol. 10(2) (2010), 637-661.

[8] M. Freedman and C. McMullen, 'Elder siblings and the taming of hyperbolic 3-manifolds', Ann. Acad. Sci. Fenn. Math. 23(2) (1998), 415-428. 
[9] A. Marden, 'The geometry of finitely generated kleinian groups', Ann. of Math. (2) 99(2) (1974), 383-462.

[10] G. D. Mostow, 'Quasi-conformal mappings in n-space and the rigidity of the hyperbolic space forms', Publ. Math. Inst. Hautes Études Sci. 43 (1968).

[11] J. Weeks, 'SnapPea, A computer program for creating and studying hyperbolic 3-manifolds', available at http://www.geometrygames.org/SnapPea.

COLIN ADAMS, Department of Mathematics and Statistics,

Williams College, Williamstown, MA 01267 USA

e-mail: Colin.C.Adams@williams.edu

KARIN KNUDSON, Department of Mathematics, University of Texas,

1 University Station C1200, Austin, TX 78712 USA

e-mail: kknudson@math.utexas.edu 